\title{
Expression of capacitation-dependent changes in chlortetracycline fluorescence patterns in mouse spermatozoa requires a suitable glycolysable substrate
}

\author{
Lynn R. Fraser and Jane E. Herod \\ Anatomy and Human Biology, Biomedical Sciences Division, King's College London, Strand, \\ London WC2R 2LS, UK
}

\begin{abstract}
Summary. Chlortetracycline (CTC) fluorescence patterns were assessed in epididymal mouse sperm suspensions capacitated in exogenous substrate-containing and substrate-free media. A capacitation-dependent transition from a majority of acrosomeintact cells expressing the uncapacitated $F$ pattern of fluorescence to a majority with the capacitated acrosome-intact B and acrosome-reacted AR patterns was confirmed for suspensions incubated a total of $120 \mathrm{~min}$ in the presence of a glycolysable substrate, glucose. In contrast, assessment of spermatozoa incubated for $120 \mathrm{~min}$ in substrate-free medium revealed a majority of cells with the uncapacitated $F$ pattern, despite an earlier demonstration that such cells are essentially capacitated: upon the introduction of glucose, suspensions are immediately highly fertile. When a suitable glycolysable substrate, either glucose or mannose but not fructose, was added to such suspensions, the distribution of CTC patterns changed within 10 min to a majority of B and AR patterns. Furthermore, the degree of change from uncapacitated to capacitated patterns was substrate concentration-dependent. In contrast, the introduction of the non-metabolizable substrates 2-deoxyglucose and 3-0-methylglucose and the oxidizable substrates sodium pyruvate and sodium lactate caused no change in the patterns from those seen in substrate-free medium. The in-vitro fertilizing ability of sperm suspensions to which increasing amounts of glucose or mannose were added, after initial substrate-free preincubation, directly paralleled the changes in CTC patterns and was as rapid as for suspensions incubated continuously in either hexose. We therefore conclude that the alteration in position of surface components to which CTC binds is not only capacitation-dependent, but also energy-dependent. In the absence of an appropriate exogenous glycolysable substrate, the final transition cannot occur, even though the cells are essentially capacitated.
\end{abstract}

Keywords: chlortetracycline; capacitation; glycolysis; acrosome reaction; mouse

\section{Introduction}

Successful mammalian fertilization requires that spermatozoa complete capacitation which permits them to express hyperactivated motility and to undergo the acrosome reaction. Numerous studies have highlighted specific ions/molecules that must be present for fertilization to occur. In some instances the component is required during both capacitation and gamete interaction, e.g. $\mathrm{Ca}^{2+}$ (reviewed by Fraser \& Ahuja, 1988). In others, the component only plays a role late in capacitation or during gamete interaction. When such molecules in question are added to spermatozoa just before mixing with eggs, fertilizing ability will be indistinguishable from that of spermatozoa incubated continuously in their presence. The mouse sperm requirement for a glycolysable substrate is a good example of this: spermatozoa preincubated in substrate-free medium and then given glucose 
are as fertile as cells incubated continuously in glucose (Fraser \& Quinn, 1981). Under substratefree conditions the acrosome reaction is inhibited and hyperactivated motility is not expressed; indeed, the motility is sluggish and erratic. However, the introduction of glucose results in rapid (within a few minutes) onset of hyperactivated motility, occurrence of spontaneous acrosome reactions and penetration of unfertilized eggs. The spermatozoa would therefore appear able to complete essentially all of capacitation in the absence of a glycolysable substrate, but such a component is required for the final stage(s) of capacitation and initiation of events culminating in sperm-egg fusion. Fusion per se does not appear to require an exogenous substrate (Fraser, 1981).

One difficulty encountered in assessing capacitation is the fact that characteristics most easily quantified, e.g. acrosome reaction, hyperactivated motility and fertilizing ability, all represent terminal events or stages. They provide little or no indication of transition to the capacitated state. However, Ward \& Storey (1984) have reported that the pattern of chlortetracycline (CTC) fluorescence observed on the mouse sperm head changes as capacitation proceeds, from a preponderance of uncapacitated acrosome-intact spermatozoa, with fluorescence over the entire head ( $F$ pattern) to a predominance of capacitated cells, either acrosome-intact with a fluorescence-free band in the postacrosomal region (B pattern) or acrosome-reacted with no fluorescence on the head (AR pattern). Therefore, it appears possible to discriminate between uncapacitated and capacitated acrosome-intact spermatozoa, a feature not offered by conventional assessment of the presence or absence of the acrosome. Since a recent report suggests that this method is also valid for evaluating human spermatozoa (Lee et al., 1987), these pattern changes may reflect alterations common to mammalian spermatozoa in general rather than just to those of the mouse.

We have used this method to assess the capacitation state of mouse sperm suspensions preincubated in the absence of added substrate. Because they respond so rapidly to the introduction of glucose (Fraser \& Quinn, 1981), we predicted that the majority of cells would exhibit the B pattern of CTC fluorescence. We also evaluated changes in the fluorescence patterns upon the introduction of increasing amounts of glucose, up to the $5.56 \mathrm{~mm}$ routinely used in complete medium, and compared these to fertilizing ability in vitro of similarly treated suspensions. Finally, we compared the CTC fluorescence patterns upon the addition of hexoses (glucose, fructose and mannose) and non-glycolysable substrates (2-deoxyglucose, 3-0-methylglucose, pyruvate and lactate) to suspensions preincubated in substrate-free medium. Neither fructose nor the non-glycolysable substrates support mouse sperm hyperactivated motility or fertilization in vitro (Fraser \& Quinn, 1981) and mannose reportedly does not support hyperactivated motility (Cooper, 1984). Since glucose supports all the events necessary for fertilization, it was felt important to compare these different substrates.

\section{Materials and Methods}

Media. The basic culture medium used for sperm incubation and fertilization in vitro was a modified Tyrode's medium (Fraser, 1983b) containing $5.56 \mathrm{~mm}$-glucose and $4 \mathrm{mg}$ bovine serum albumin (BSA, crystalline; Sigma Chemical Co., Poole, Dorset. UK) per ml; neither lactate nor pyruvate were present. Substrate-free medium was prepared by omitting the glucose. Concentrated glucose and mannose stock solutions, in Tyrode's medium, were prepared so that $10 \mu \mathrm{l}$ appropriate stock added to $240 \mu \mathrm{l}$ substrate-free medium would produce the following final concentrations of hexose: $5.56 \mu \mathrm{M}, 55.6 \mu \mathrm{M}, 278 \mu \mathrm{M}, 556 \mu \mathrm{M}$ and $5.56 \mathrm{~mm}$. Stock solutions to produce $5.56 \mathrm{~mm}$ fructose, 2-deoxyglucose and 3-0-methylglucose and $0.5 \mathrm{~mm}$-sodium pyruvate were also prepared. Sodium lactate syrup was added to give a final concentration of $20 \mathrm{~mm}$.

Sperm incubation. Spermatozoa from the cauda epididymidis were obtained from mature ( $>8$ weeks) TO male mice (Harlan OLAC, Bicester, Oxon, UK). The gametes were released, by extrusion using 2 pairs of sterile watchmakers forceps, into medium ( $1 \mathrm{ml}$ per each pair of epididymides) in a sterile $30-\mathrm{mm}$ plastic culture dish (Sterilin, Teddington, Middlesex, UK) overlaid with autoclaved liquid paraffin (Boots, Nottingham, UK). Suspensions were placed in an anaerobic culture jar, gassed with a mixture of $5 \% \mathrm{CO}_{2}-5 \% \mathrm{O}_{2}-90 \% \mathrm{~N}_{2}$ and incubated at $37^{\circ} \mathrm{C}$ for a total of $120 \mathrm{~min}$. 
In the first experimental series, a suspension was prepared in glucose-containing medium. After 10 , 60 and $120 \mathrm{~min}$, aliquants were removed and assessed for capacitation state using the CTC fluorescence method described below. Three replicates were carried out $(n=3)$.

In the second series, sperm suspensions $(n=4)$ were prepared in substrate-free medium $(-\mathrm{G})$; an aliquant was removed and appropriate glucose stock solution was added to produce a final concentration of $5.56 \mathrm{~mm}$-glucose $(+G)$. These 2 suspensions were incubated for $120 \mathrm{~min}$; a second aliquant was removed from the substrate-free suspension and glucose stock solution was added to produce $5.56 \mathrm{~mm}$-glucose $(-\mathrm{G} \rightarrow+\mathrm{G})$. After $10 \mathrm{~min}$, all 3 suspensions were assessed using CTC fluorescence.

In the third series, sperm suspensions $(n=4)$ were prepared in substrate-free medium and incubated for 120 min. Four aliquants were removed and to each was added the appropriate stock solution to produce $5 \cdot 56 \mu \mathrm{M}-, 55 \cdot 6 \mu \mathrm{M}-$, $556 \mu \mathrm{M}$ - and $5.56 \mathrm{~mm}$-glucose. After $10 \mathrm{~min}$, the glucose-free and giucose-containing suspensions were assessed using CTC fluorescence.

In the fourth series, sperm suspensions $(n=3)$ were prepared and incubated for 120 min in substrate-free medium. Three aliquants were removed and to each was added the appropriate stock solution to produce $5.56 \mathrm{~mm}$-glucose, -mannose and -fructose. After 10 min these and the substrate-free suspension were assessed using CTC fluorescence.

In the fifth series, sperm suspensions $(n=3)$ were prepared and incubated for $120 \mathrm{~min}$ in substrate-free medium. Five aliquants were removed and to each was added the appropriate stock solution to produce $5.56 \mathrm{~mm}$-glucose, -2-deoxyglucose and -3-0-methylglucose, $0.5 \mathrm{~mm}$-sodium pyruvate and 20 mm-sodium lactate. After $10 \mathrm{~min}$ these and the substrate-free suspension were assessed using CTC fluorescence.

In-vitro fertilization. Unfertilized cumulus-intact eggs were obtained from mature ( $>8$ weeks) TO female mice induced to superovulate by injection of 7.5 i.u. PMSG (Pregnyl: Organon, Morden, Surrey, UK) and, $54 \mathrm{~h}$ later, 5 i.u. hCG (Gestyl: Organon). Sperm suspensions $(n=3)$ were prepared as for the second experimental series above in substrate-free, $5.56 \mathrm{~mm}$-glucose-containing and $5.56 \mathrm{~mm}$-mannose-containing media and incubated for $120 \mathrm{~min}$. These were then diluted 10-fold into media containing various amounts of glucose or mannose. Suspensions incubated continuously in glucose/mannose were diluted into glucose/mannose-containing medium. Those incubated in the absence of substrate were diluted into medium containing $0,5.56 \mu \mathrm{M}, 55.6 \mu \mathrm{M}, 228 \mu \mathrm{M}, 556 \mu \mathrm{M}$ and $5.56 \mathrm{mM}-$ glucose and $55.6 \mu \mathrm{M}, 556 \mu \mathrm{M}$ and $5.56 \mathrm{~mm}$-mannose. A $400 \mu \mathrm{l}$ droplet of diluted suspension was added to a plastic culture dish containing sterile liquid paraffin. Recently ovulated cumulus-intact eggs ( $14 \mathrm{~h}$ after hCG) were added; after gassing with $5 \% \mathrm{CO}_{2}-5 \% \mathrm{O}_{2}-90 \% \mathrm{~N}_{2}$, gametes were incubated for $65 \mathrm{~min}$.

Eggs were then transferred to a droplet of fresh medium and fixed at 75 min after gamete mixing by flooding the dishes with buffered formalin ( $\mathrm{pH} 7 \cdot 4,4 \%$ formaldehyde). After a minimum of 30 min, eggs were stained with $0 \cdot 75 \%$ orcein in $45 \%$ glacial acetic acid and assessed for the presence of a fertilizing sperm head. The stage in meiosis II reached by the egg chromosomes and the degree of sperm head decondensation were used to gauge the rapidity of sperm penetration and gamete fusion. Rapid gamete interaction, characteristic of fully capacitated suspensions, results in a high proportion of eggs at the telophase II-second polar body stage with fully decondensed sperm heads after $75 \mathrm{~min}$ incubation (Fraser, 1983a).

CTC fluorescence assay. A modification of the Ward \& Storey (1984) method was used. CTC (Sigma) at $750 \mu \mathrm{M}$ was prepared in a buffer of $20 \mathrm{~mm}$-Tris, $130 \mathrm{~mm}-\mathrm{NaCl}$ and $5 \mathrm{~mm}$-cysteine, final $\mathrm{pH} 7 \cdot 8$. The sperm suspension $(5 \mu \mathrm{l})$ was mixed with $5 \mu \mathrm{l} \mathrm{CTC}$ solution on a clean warm slide; after $30 \mathrm{sec}, 0.2 \mu \mathrm{l} 12.5 \%$ paraformaldehyde in $0.5 \mathrm{~m}$-Tris buffer (final pH 7.4) was added and mixed well. Finally, a drop of 0.22 M-1,4-diaza-bicyclo (2,2,2)-octane (DABCO; Sigma) in glycerol was mixed in to retard fading of fluorescence. After adding a clean coverslip, the slide was gently compressed using a tissue. This helped to flatten the spermatozoa so that accurate assessment could be made. Slides were kept in a light-shielded container with a moist environment until they could be examined using an Olympus BHS microscope equipped with phase-contrast and epifluorescence optics. The excitation beam was passed through a $405 \mathrm{~nm}$ band pass filter and CTC fluorescence emission was observed through a DM 455 dichroic mirror.

In each aliquant 100 sperm heads, 50 on each of 2 slides, were classified as having 1 of 3 staining patterns: (1) ' $F$ ' with fuorescence over the entire head, characteristic of acrosome-intact uncapacitated spermatozoa; (2) 'B' with a fluorescence-free band in the postacrosomal region characteristic of acrosome-intact capacitated spermatozoa; (3) 'AR' with fluorescence absent from the head, characteristic of acrosome-reacted spermatozoa. The presence or absence of the acrosomal cap was verified using phase-contrast illumination (Fraser, 1983b). It was noticed that some cells devoid of the acrosomal cap, as determined by phase contrast, presented a CTC pattern more closely resembling $\mathrm{B}$ than AR, i.e. fluorescence was still present in the acrosomal region. We reasoned that these had recently started the membrane-fusion events associated with the acrosome reaction and that total loss of CTC-binding material had not yet occurred. Because these were lacking a detectable acrosomal cap, they were counted as AR cells. Fluorescence of the midpiece was noted under all conditions used.

Because the technique utilizes an unselected population of spermatozoa, after incubation for 120 min a certain proportion of cells will be non-motile, hence potentially dead and possibly degenerating. Therefore, at least some of the cells scored as AR will not necessarily have undergone a physiological acrosome reaction. When suspensions are treated to select motile cells, e.g. by filtration (Fraser, 1983b), the background level of acrosomeless spermatozoa will be $\leqslant 10 \%$; with the present method, it is probably nearer $20 \%$.

Statistical analysis. Data were analysed using Cochran's modification of $2 \times 2$ contingency tables (Snedecor \& Cochran, 1967). Since this method uses within-replicate comparisons, consistent responses and an adequate number of gametes assessed are required to obtain significant differences. 


\section{Results}

\section{Series I: CTC fluorescence patterns in glucose-containing medium}

When suspensions were incubated for a total of $120 \mathrm{~min}$ in the presence of glucose, the CTC fluorescence patterns changed in a manner consistent with that reported by Ward \& Storey (1984). After $10 \mathrm{~min}$, the great majority of cells exhibited the $\mathrm{F}$ pattern of acrosome-intact uncapacitated spermatozoa (Fig. 1). As incubation time increased, the proportion of cells with the uncapacitated $F$ pattern decreased and that with the capacitated B and AR patterns increased; after 120 min the majority of cells were exhibiting a capacitated pattern. Significant differences between 10 and 120 min incubation were observed for all 3 patterns $(P<0.05-P<0.001)$ and between 60 and 120 min for $\mathrm{F}(P<0.05)$ and AR $(P<0.025)$ patterns. The changes in CTC fluorescence, with a majority of cells capacitated after $120 \mathrm{~min}$ incubation in control medium, are therefore consistent with the demonstration that similarly-incubated suspensions are highly fertile (e.g. Fraser, 1983a, b; Table 1, this study).

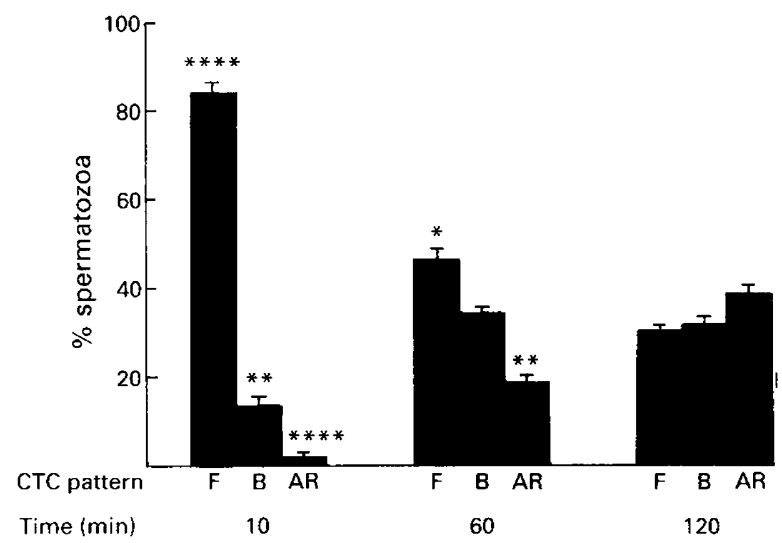

Fig. 1. CTC fluorescence patterns in mouse sperm suspensions incubated in $5.56 \mathrm{~mm}$-glucosecontaining medium. Samples were removed at 10,60 and $120 \mathrm{~min}$ and assessed for the 3 different patterns. Data are the mean of 3 replicates \pm s.e.m. ${ }^{*} P<0.05,{ }^{* *} P<0.025$, $* * * * P<0.001$ compared with values for 120 min suspensions.

\section{Series II: CTC fluorescence in the absence/presence of glucose}

When suspensions were incubated for $120 \mathrm{~min}$ in the absence of glucose, the CTC patterns, with a majority of cells expressing the $\mathrm{F}$ pattern, differed markedly from those observed in glucoseincubated spermatozoa (Fig. 2). Significant differences were observed for all 3 patterns: F, $P<0.01 ; \mathrm{B}, P<0.05 ; \mathrm{AR}, P<0.025$. The distribution in the substrate-free suspensions resembled that seen in spermatozoa incubated for $10 \mathrm{~min}$ in glucose-containing medium (Fig. 1), while that in the glucose-containing suspensions was very similar to the one seen in 120 min-incubated suspensions in Series I. When glucose was added to the substrate-free suspensions, the patterns observed were not significantly different from those seen in continuous glucose. The addition of glucose therefore caused a rapid shift in the CTC patterns from those typical of an uncapacitated suspension incubated for a short time in the presence of glucose to those typical of a capacitated suspension incubated for $120 \mathrm{~min}$ in glucose. This therefore suggested that, despite the CTC patterns expressed in substrate-free conditions, the spermatozoa were in fact capacitated and able to respond by changing patterns rapidly once an exogenous substrate was available. 


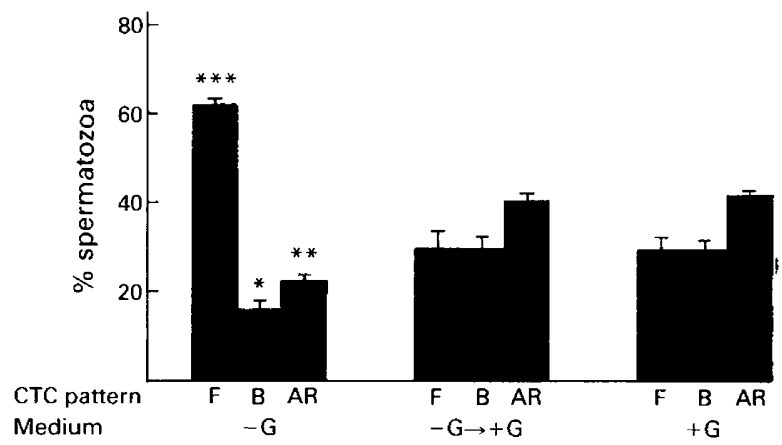

Fig. 2. CTC fluorescence patterns in mouse sperm suspensions incubated for $120 \mathrm{~min}$ in substrate-free $(-G)$ or $5.56 \mathrm{~mm}$-glucose-containing $(+G)$ medium. At $120 \mathrm{~min}$ a sample was removed from the $-G$ suspension and received $5.56 \mathrm{~mm}$-glucose $(-G \rightarrow+G)$. After $10 \mathrm{~min}$, all suspensions were assessed. Data are the mean of 4 replicates \pm s.e.m. ${ }^{*} P<0.05$, ${ }^{* *} P<0.025,{ }^{* * *} P<0.01$ compared with $+\mathrm{G}$ suspensions.

\section{Series III: CTC fluorescence in increasing concentrations of glucose}

When suspensions were incubated for $120 \mathrm{~min}$ in substrate-free medium and then received increasing concentrations of glucose for $10 \mathrm{~min}$, a concentration-dependent change in the CTCfluorescence patterns was observed (Fig. 3). In millimolar glucose, the distribution was like that seen in similarly treated suspensions in Series II (see Fig. 2), with the majority of cells expressing a capacitated pattern. Significant differences between these suspensions and those receiving $0-55.6 \mu \mathrm{M}$-glucose were observed for some or all of the 3 patterns $(P<0.05-P<0.01$, Fig. 3); in the suspensions with low glucose, the majority of cells were expressing the uncapacitated $F$ pattern. While no obvious differences were noted between 0 and $5.56 \mu \mathrm{M}$-glucose, the introduction of $55.6 \mu \mathrm{M}$-glucose caused a shift towards the capacitated patterns. This shift was more marked in $556 \mu \mathrm{M}$-glucose and the distribution of patterns in this concentration did not differ significantly from that observed with $5.56 \mathrm{~mm}$-glucose. When the proportion of cells expressing each pattern was plotted against increasing amounts of glucose added after $120 \mathrm{~min}$, a linear decrease in the $\mathrm{F}$ pattern and linear increases in the B and AR patterns were revealed (Fig. 4). These results confirm that the response to exogenous substrate is rapid and demonstrate that it is concentrationdependent.

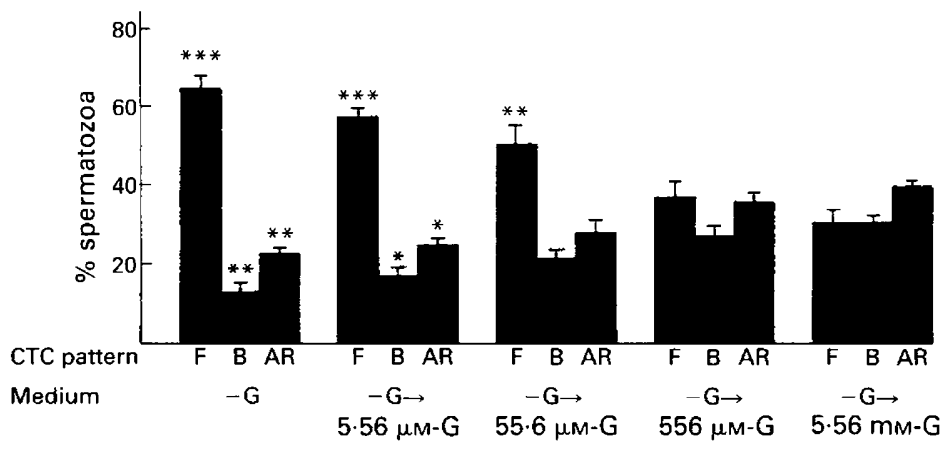

Fig. 3. CTC fluorescence patterns in mouse sperm suspensions incubated in substrate-free $(-\mathrm{G})$ medium for $120 \mathrm{~min}$ and then receiving glucose to give final concentrations of: $5.56 \mu \mathrm{M}$, $55.6 \mu \mathrm{M}, 556 \mu \mathrm{M}$ and $5.56 \mathrm{~mm}$. After $10 \mathrm{~min}$, all suspensions were assessed. Data are the mean of 4 replicates \pm s.e.m. ${ }^{*} P<0.05,{ }^{* *} P<0.025,{ }^{* * *} P<0.01$ compared with $-\mathrm{G} \rightarrow 5.56 \mathrm{~mm}$ glucose suspensions. 


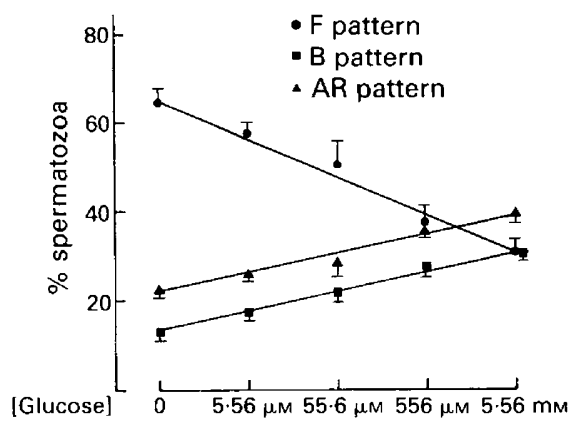

Fig. 4. Glucose concentration-dependent changes in the distribution of CTC fluorescence patterns F, B and AR. Data are from experiments presented in Fig. 3, with percentage of spermatozoa in each category plotted against glucose concentration. Data are mean of 4 replicates \pm s.e.m.

\section{Series IV: CTC fluorescence in the presence of various hexoses}

When suspensions incubated for $120 \mathrm{~min}$ in substrate-free medium received $5.56 \mathrm{~mm}$-glucose, -mannose or -fructose, the CTC fluorescence patterns varied, depending on the specific hexose provided. As in the other series, the majority of cells in substrate-free conditions exhibited the uncapacitated $F$ pattern, while those receiving glucose had a majority of capacitated cells (Fig. 5). Significant differences between these conditions were observed for all 3 patterns $(P<0.025-$ $P<0.001)$. When mannose was added, the distribution of CTC patterns was very similar to that observed in the presence of glucose with no significant differences between the two groups. However, when fructose was added, the distribution of CTC patterns more closely resembled the substrate-free suspensions than the glucose-containing ones. Significant differences in the proportions of cells with the $\mathrm{F}(P<0.01)$ and $\mathrm{AR}(P<0.05)$ patterns were noted when fructose was compared with glucose. When substrate-free conditions were compared with the presence of fructose, there was a lower incidence $(P<0.05)$ in $\mathrm{F}$ pattern cells in fructose, but no significant differences were noted for the B and AR patterns. Therefore, our data indicate that mannose can support the acrosome reaction to the same extent as glucose, but that fructose cannot.

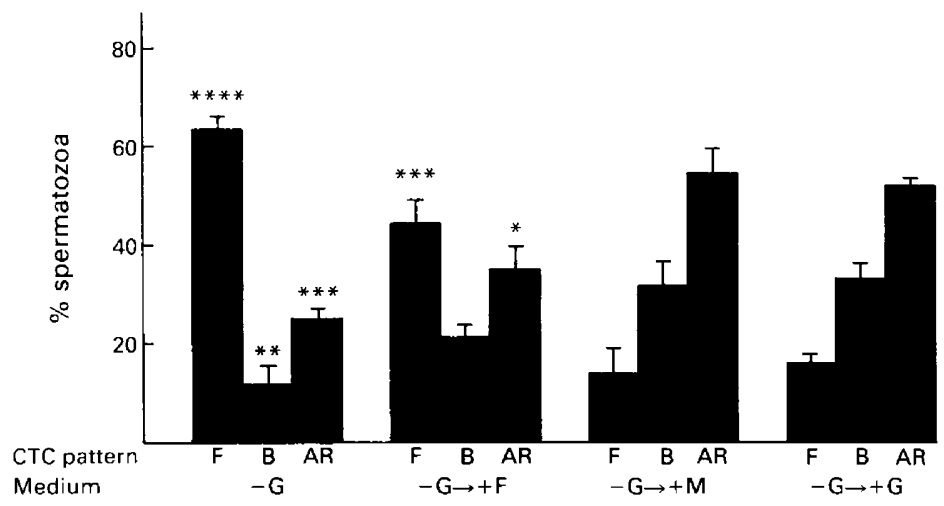

Fig. 5. CTC fluorescence patterns in mouse sperm suspensions incubated in substrate-free $(-\mathrm{G})$ medium for $120 \mathrm{~min}$ and then receiving $5.56 \mathrm{~mm}$-fructose $(-\mathrm{G} \rightarrow+\mathrm{F})$, mannose $(-G \rightarrow+M)$ or glucose $(-G \rightarrow+G)$. After $10 \mathrm{~min}$, all suspensions were assessed. Data are the mean of 3 replicates \pm s.e.m. ${ }^{*} P<0.05,{ }^{* *} P<0.025,{ }^{* * *} P<0.01,{ }^{* * * *} P<0.001$ compared with $-\mathrm{G} \rightarrow+\mathrm{G}$ suspensions. 
In this series, and Series V, the overall distribution of F, B and AR patterns in the presence of glucose differed from that seen in Figs $1-3$, with fewer $F$ and more AR cells. This presumably reflects differences in suspensions, since a gap of several months occurred between Series I-III and Series IV and V.

\section{Series V: CTC patterns in the presence of non-glycolysable substrates}

After incubation for $120 \mathrm{~min}$ in substrate-free medium, the distribution of CTC patterns was as seen previously: a majority of cells exhibited the $\mathrm{F}$ pattern (Fig. 6). This same distribution was observed in suspensions which received 2-deoxyglucose, 3-0-methylglucose, pyruvate or lactate. Only the introduction of glucose caused a shift to predominantly capacitated cells and significant changes in the proportions of cells exhibiting the 3 different patterns $(P<0.05-P<0.001$; Fig. 6).

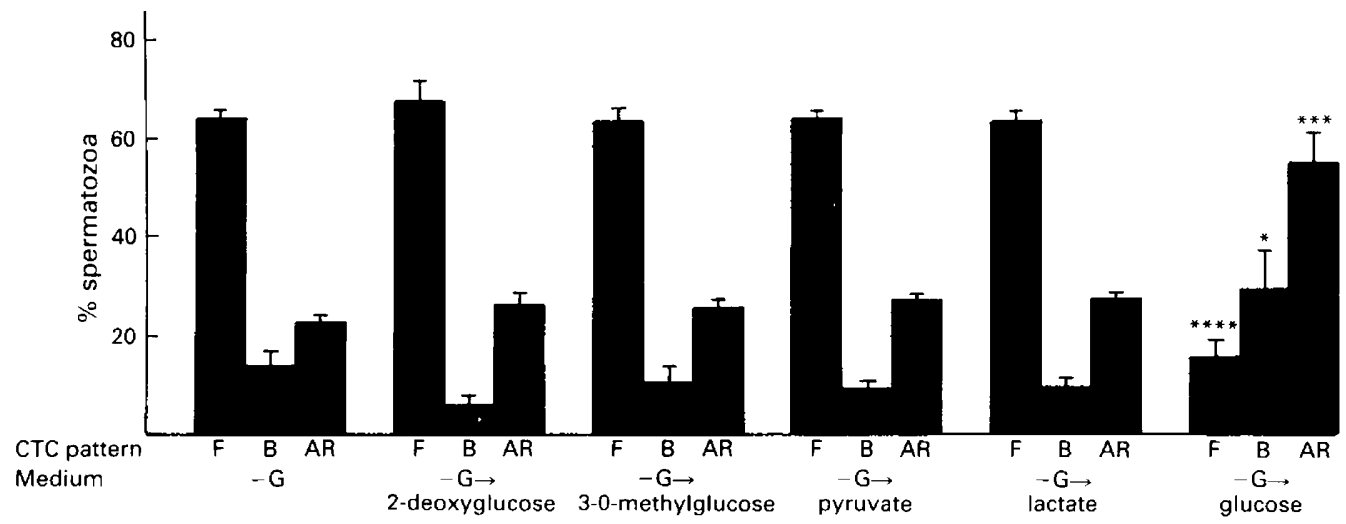

Fig. 6. CTC fluorescence patterns in mouse sperm suspensions incubated in substrate-free ( $-\mathrm{G})$ medium for $120 \mathrm{~min}$ and then receiving $5.56 \mathrm{~mm}$-glucose, 2-deoxyglucose, 3-0-methylglucose, $20 \mathrm{~mm}$-lactate or $0.5 \mathrm{~mm}$-pyruvate. After $10 \mathrm{~min}$, all suspensions were assessed. Data are the mean of 3 replicates \pm s.e.m. ${ }^{*} P<0.05,{ }^{* *} P<0.01,{ }^{* * *} P<0.001$ compared with substrate-free suspensions.

\section{Series VI: fertilizing ability in increasing concentrations of glucose and mannose}

When sperm suspensions were preincubated in glucose-free medium and received increasing concentrations of hexose, their ability to fertilize cumulus-intact eggs was dependent on the concentration of glucose or mannose provided (Table 1). Control suspensions incubated continuously in glucose were highly fertile, with $91.1 \%$ of eggs being fertilized. However, when suspensions preincubated in the absence of glucose received $5.56 \mu \mathrm{M}$-glucose just before introduction of eggs, no eggs were fertilized. With the addition of 55.6 and $278 \mu \mathrm{M}$-glucose, some fertilization was observed (20.9\% and $37.0 \%$, respectively); these values were significantly lower $(P<0.01)$ than those obtained in the $5.56 \mathrm{~mm}$-glucose suspensions. With the addition of $556 \mu \mathrm{M}$ - and $5.56 \mathrm{~mm}$-glucose, a high proportion of eggs was fertilized $(82.4 \%$ and $95.3 \%$, respectively), results statistically similar to those obtained in continuous glucose.

Responses in the presence of increasing amounts of mannose were similar. Sperm suspensions were highly fertile in the continuous presence of $5.56 \mathrm{~mm}$-mannose with $95.5 \%$ of eggs fertilized (Table I). After 120 min substrate-free preincubation, the addition of $55.6 \mu \mathrm{M}$-mannose resulted in only $21.9 \%$ of eggs being fertilized, significantly fewer $(P<0.01)$ than in the presence of $5.56 \mathrm{mm-}$ mannose. In contrast, the introduction of $556 \mu \mathrm{M}$ - or $5.56 \mathrm{~mm}$-mannose resulted in $89.3 \%$ and $87.9 \%$, respectively, of eggs being fertilized, values that did not differ significantly from that for continuous mannose. 
Table 1. Hexose concentration-dependence of mouse sperm fertilizing ability

\begin{tabular}{|c|c|c|c|c|c|c|}
\hline \multirow[b]{2}{*}{ Hexose } & \multicolumn{2}{|c|}{ Hexose concentration } & \multicolumn{3}{|c|}{ Eggs fertilized } & \multirow{2}{*}{$\begin{array}{c}\text { Rapid gamete } \\
\text { interaction } \dagger \\
\%\end{array}$} \\
\hline & Preincubation & Fertilization & No. & $\%$ & (range) & \\
\hline Glucose & $\begin{array}{c}5.56 \mathrm{mM} \\
0\end{array}$ & $\begin{array}{c}5.56 \mathrm{mM} \\
5.56 \mu \mathrm{M} \\
55.6 \mu \mathrm{M} \\
278 \mu \mathrm{M} \\
556 \mu \mathrm{M} \\
5.56 \mathrm{mM}\end{array}$ & $\begin{array}{c}92 / 101 \\
0 / 55 \\
9 / 43 \\
20 / 54 \\
61 / 74 \\
82 / 86\end{array}$ & $\begin{array}{c}91 \cdot 1 \\
0 \\
20 \cdot 9 \\
37 \cdot 0 \\
82 \cdot 4 \\
95 \cdot 3\end{array}$ & $\begin{array}{c}(87-100) \\
-* * * * \\
(0-47)^{* * *} \\
(26-63)^{* * *} \\
(73-100) \\
(87-100)\end{array}$ & $\begin{array}{c}96 \cdot 7 \\
\overline{44 \cdot 4} \\
65 \cdot 0 \\
80 \cdot 3 \\
96 \cdot 3\end{array}$ \\
\hline Mannose & $\begin{array}{c}5.56 \mathrm{mM} \\
0\end{array}$ & $\begin{array}{c}5.56 \mathrm{mM} \\
55.6 \mu \mathrm{M} \\
556 \mu \mathrm{M} \\
5.56 \mathrm{mM}\end{array}$ & $\begin{array}{r}63 / 66 \\
9 / 41 \\
51 / 58 \\
67 / 75\end{array}$ & $\begin{array}{l}95.5 \\
21.9 \\
87.9 \\
89.3\end{array}$ & $\begin{array}{l}(93-100) \\
(14-26)^{* * *} \\
(79-100) \\
(75-100)\end{array}$ & $\begin{array}{l}92 \cdot 1 \\
44 \cdot 4 \\
94 \cdot 1 \\
97 \cdot 0\end{array}$ \\
\hline
\end{tabular}

†Fertilized eggs at telophase II-second polar body stage with fully decondensed sperm head.

The data for 3 replicates are presented as the no. and \% of eggs fertilized/total no. of eggs examined and the range of $\%$ eggs fertilized in individual replicates. ${ }^{* * *} P<0.01,{ }^{* * * *} P<0.001$ compared with continuous $5.56 \mathrm{~mm}$ appropriate hexose.

When the incidence of fertilization was reduced, gamete interactions were also less rapid as evidenced by fewer eggs at telophase II-second polar body with fully decondensed sperm heads. This reflects not only the reduced proportion of cells showing a capacitated pattern of CTC fluorescence (Figs $3 \& 4$ ) but also a reduction in the proportion of cells expressing hyperactivated motility. In the absence of substrate, spermatozoa were poorly motile with erratic movements (see also Fraser \& Quinn, 1981). The introduction of $5.56 \mu \mathrm{M}$-glucose was sufficient to improve motility but no hyperactivated motility could be detected. This would be consistent with the total failure to achieve fertilization under these conditions. With increasing amounts of glucose or mannose, hyperactivated motility was observed (subjective assessment) in $\sim 10 \%$ of cells at $55.6 \mu \mathrm{M}$-hexose up to $\sim 50 \%$ of motile cells at $5.56 \mathrm{~mm}$-glucose or mannose.

\section{Discussion}

Our data provide evidence that the changes in CTC fluorescence patterns involve energy-dependent as well as capacitation-dependent events. We first confirmed the observations of Ward \& Storey (1984) that, under conditions supporting complete capacitation and fertilization in vitro (Fraser, 1983a), there is a time-dependent change in the distribution of the 3 CTC patterns of F, B and AR. In uncapacitated suspensions, the majority of cells exhibit the uniform fluorescence $F$ pattern, while in capacitated and fully fertile suspensions the majority of cells exhibit the capacitated B and AR patterns. In the TO mice used here there is a clear increase in the incidence of spontaneous acrosome reactions as capacitation proceeds, an observation consistent with the data of Ward \& Storey (1984) in the original description of the CTC assessment method. We then examined CTC fluorescence patterns in sperm suspensions incubated in the absence of exogenous energy substrate. In an earlier study demonstrating that cells incubated in this medium could rapidly switch on hyperactivated motility, undergo spontaneous acrosome reactions and fertilize eggs, all within a few minutes of glucose addition, we had concluded that these spermatozoa were essentially fully capacitated (Fraser \& Quinn, 1981). If not, there would have been a detectable lag between introduction of glucose and onset of these features as has been observed following the introduction of $\mathrm{Ca}^{2+}$ to $\mathrm{Ca}^{2+}$-deficient suspensions when a further $1 \mathrm{~h}$ is required to complete capacitation (see Fraser \& Ahuja, 1988). In the present study we therefore predicted that the majority of cells would exhibit the capacitated but acrosome-intact B pattern. However, what we actually observed was a majority of cells with the $\mathrm{F}$ pattern and an overall distribution characteristic of uncapacitated 
sperm suspensions incubated for a short time in complete medium (Fig. 1). The fact that the pattern altered, within a few minutes of glucose introduction, to a distribution characteristic of capacitated fertilizing sperm suspensions (Figs $1 \& 2$ ) led us to conclude that the change in distribution of surface molecules revealed by CTC fluorescence is an energy-dependent process. In the absence of a suitable energy substrate, surface molecules remain in their original positions even if the cells have changed in terms of functional potential and are essentially capacitated.

The introduction of increasing amounts of glucose to suspensions preincubated in substratefree medium revealed a clear concentration-dependence in the distribution of CTC patterns. The majority of cells exhibited the $F$ pattern when the glucose concentration was $\leqslant 55.6 \mu \mathrm{M}$, with very similar values for 0 and $5.56 \mu \mathrm{M}$. No significant differences were noted among the patterns in the 2 highest glucose concentrations assessed, i.e. $556 \mu \mathrm{M}$ and $5.56 \mathrm{mM}$, with B and AR patterns predominating. From these responses one might predict that the fertilizing ability of such suspensions would differ, with possibly the two lowest being non-fertilizing, the two highest being highly fertile and the middle one $(55.6 \mu \mathrm{M})$ being in between.

When the in-vitro fertilizing ability of sperm suspensions treated in the above manner was investigated, the data obtained were consistent with our predictions. Because the greatest change in proportion of F-pattern cells occurred between 55.6 and $556 \mu \mathrm{M}$-glucose, the intermediate concentration of $278 \mu \mathrm{M}$ was also evaluated. We observed no fertilization in 0 or $5.56 \mu \mathrm{M}$, some $(21 \%)$ in $55.6 \mu \mathrm{M}$, rather more $(37 \%)$ in $278 \mu \mathrm{M}$ and high levels in $556 \mu \mathrm{M}$ and $5.56 \mathrm{~mm}$-glucose $(82 \%$ and $95 \%$, respectively). The latter two did not differ statistically either from each other or from continuous glucose-incubated suspensions (91\%; Table 1). Thus the change in CTC patterns reflected the change in demonstrable fertilizing ability.

Successful fertilization requires hyperactivated motility as well as the acrosome reaction (Fraser, 1981) and this pattern of motility is energy substrate-dependent (Fraser \& Quinn, 1981; Cooper, 1984). In the present study, the concentration-dependence of motility changes paralleled that of the CTC pattern distribution, with $556 \mu \mathrm{M}$-glucose being sufficient to support both to a similar extent as seen in the standard $5.56 \mathrm{~mm}$-glucose-containing medium. Although Cooper (1984) reported that mannose did not support hyperactivated motility, we observed it in these suspensions. While our assessments were subjective and Cooper (1984) used a more objective photographic method, our data clearly indicate that mannose also supports the acrosome reaction and fertilization in vitro in a manner indistinguishable from glucose. One possible explanation could be the use of a high ionic strength medium by Cooper (1984) to shorten capacitation time, while a standard ionic strength medium was used in the present study. Perhaps, under the former conditions, spermatozoa differ in their responses to glucose and mannose.

The fact that introduction of the non-metabolizable compounds 2-deoxyglucose and 3-0methylglucose and the oxidizable substrates pyruvate and lactate, all of which failed to support fertilization (Fraser \& Quinn, 1981), failed to alter the CTC patterns from those observed in the absence of substrate suggests strongly that a glycolysable compound is required for transition from the $\mathrm{F}$ to the $\mathrm{B}$ and AR patterns. The present study has demonstrated that both glucose and mannose are suitable glycolysable substrates for all the changes required for fertilization. The observation of a consistent decline in $\mathrm{O}_{2}$ consumption by mouse spermatozoa during capacitation (Fraser \& Lane, 1987), together with the requirement for a glycolysable substrate, suggests a switch from oxidative phosphorylation to glycolysis as cells reach functional ability. The inability of fructose to support the transition to B and AR patterns was consistent with earlier studies indicating that fructose was unable to support fertilization in the mouse (Hoppe, 1976; Fraser \& Quinn, 1981) and the rat (Niwa \& Iritani, 1978). The facts that mouse spermatozoa can glycolyse fructose, albeit to a lesser extent than glucose (Hoppe, 1976), and that $\mathrm{O}_{2}$ consumption is consistently low throughout capacitation (Fraser \& Lane, 1987), suggest that deficiencies in transport and/or intracellular metabolism lead to insufficient energy production from fructose.

At present the molecular species to which CTC binds is unclear, although it is presumably located on the plasma membrane. We have obtained evidence that the CTC patterns reflect the 
presence or absence of a surface-associated decapacitation factor derived from the epididymis (Fraser et al., 1990). In an earlier study it was demonstrated that this factor can be removed by gentle centrifugation: washing of poorly fertile sperm suspensions and resuspension in fresh medium resulted in cells with fertilization kinetics resembling those of fully capacitated spermatozoa (Fraser, 1984). In contrast, the re-introduction of decapacitation factor-containing sperm washings to capacitated cells rendered them poorly, although reversibly, fertile. We now have demonstrated that the CTC patterns expressed by sperm suspensions treated in these ways are altered in a manner consistent with their demonstrable functional state. The removal of decapacitation factor resulted in a rapid transition from a majority of uncapacitated F-pattern cells to a majority of capacitated B- and AR-pattern cells. The re-introduction of the factor inhibited the acrosome reaction and resulted in a majority of acrosome-intact cells with the F pattern of CTC fluorescence (Fraser et al., 1990). The factor appears to have a finite physiological lifespan: inhibitory activity detected in sperm washings is significantly lower in capacitated than in uncapacitated suspensions (Fraser, 1984), suggesting that the factor is either inactivated or destroyed during capacitation. Given that the present study demonstrates that the transition from the $F$ to $B$ patterns occurs rapidly, as does fertilizing ability, inactivation of the decapacitation factor would appear to occur in the absence of an exogenous energy supply but the final alteration in position would appear to be energy-dependent.

At present it is not possible to determine whether the CTC is actually binding to the decapacitation factor on the sperm surface, although the F pattern, with fluorescence over the entire head, predominates when active factor is present and the B pattern, with fluorescence lacking in the postacrosomal region, predominates when factor is reduced or absent. The transition from $F$ to $B$ pattern could represent either migration of the CTC-binding component away from the postacrosomal region or its loss from the spermatozoon. The possibility of migration is reminiscent of the situation in guinea-pig spermatozoa, in which the antigen $\mathrm{PH}-20$ has been shown to migrate from the postacrosomal region to the inner acrosomal membrane during the acrosome reaction (Myles \& Primakoff, 1984). However, in mouse spermatozoa CTC fluorescence is absent from the acrosomal region of AR pattern cells, indicating eventual loss of the CTC-binding molecules.

In conclusion, the present study has demonstrated that the transition from the $F$ to $B$ and AR patterns of CTC binding is energy-dependent, as well as capacitation-dependent. A glycolysable substrate is required to supply this energy requirement, with glucose and mannose but not fructose being suitable hexoses. Both these transitions and demonstrable fertilizing ability are substrate concentration-dependent, with $\geq 556 \mu \mathrm{M}$ glucose or mannose being sufficient to fulfil the requirements. These results also demonstrate that the distribution of CTC binding patterns does not necessarily provide an unequivocal method for assessment of capacitation state: a coupling of the CTC method with functional assessments of gametes provides a more reliable approach.

This study was supported in part by a grant from the Medical Research Council. J.H. was an Intercalated B.Sc. student. We thank Andrew Osborne for the original artwork.

\section{References}

Cooper, T.G. (1984) The onset and maintenance of hyperactivated motility of spermatozoa from the mouse. Gamete Res. 9, 55-74.

Fraser, L.R. (1981) Dibutyryl cyclic AMP decreases capacitation time in vitro in mouse spermatozoa. $J$. Reprod. Fert. 62, 63-72.

Fraser, L.R. (1983a) Mouse sperm capacitation assessed by kinetics and morphology of fertilization in vitro. $J$. Reprod. Fert. 69, 419-428.

Fraser, L.R. (1983b) Potassium ions modulate expression of mouse sperm fertilizing ability, acrosome reaction and whiplash motility in vitro. $J$. Reprod. Fert. 69, 539-553.

Fraser, L.R. (1984) Mouse sperm capacitation in vitro involves loss of a surface-associated inhibitory component. J. Reprod. Fert. 72, 373-384.

Fraser, L.R. \& Ahuja, K.K. (1988) Metabolic and surface events in fertilization. Gamete Res. 20, 491-519.

Fraser, L.R. \& Lane, M.R. (1987) Capacitation- and fertilization-related alterations in mouse sperm oxygen consumption. J. Reprod. Fert. 81, 385-393. 
Fraser, L.R. \& Quinn, P.J. (1981) A glycolytic product is obligatory for initiation of the sperm acrosome reaction and whiplash motility required for fertilization in the mouse. J. Reprod. Fert. 61, 25-35.

Fraser, L.R., Harrison, R.A.P. \& Herod, J.E. (1990) Characterization of a decapacitation factor associated with epididymal mouse spermatozoa. J. Reprod. Fert. 89, (in press).

Hoppe, P.C. (1976) Glucose requirement for mouse sperm capacitation in vitro. Biol. Reprod. 15, 3945.

Lee, M.A., Trucco, G.S., Bechtol, K.B., Wummer, N., Kopf, G.S., Blasco, L. \& Storey, B.T. (1987) Capacitation and acrosome reactions in human spermatozoa monitored by a chlortetracycline fluorescence assay. Fert. Steril. 48, 649-658.
Myles, D.G. \& Primakoff, P. (1984) Localized surface antigens of guinea pig sperm migrate to new regions prior to fertilization. J. Cell Biol. 99, 1634-1641.

Niwa, K. \& Iritani, A. (1978) Effect of various hexoses on sperm capacitation and penetration of rat eggs in vitro. J. Reprod. Fert. 53, 267-271.

Snedecor, G. \& Cochran, W. (1967) Statistical Methods, 6th edn. Iowa State University Press, Ames.

Ward, C.R. \& Storey, B.T. (1984) Determination of the time course of capacitation in mouse spermatozoa using a chlortetracycline fluorescence assay. Devl Biol. 104, 287-296.

Received 24 July 1989 